\title{
Novel subjet observables for jet quenching in heavy-ion collisions
}

\author{
Liliana Apolinário $^{1,2, a}{ }^{\mathbb{D}}$, José Guilherme Milhano ${ }^{1,2,3, \mathrm{~b}}$, Mateusz Ploskon ${ }^{4, \mathrm{c}}$, XiaomingZhang ${ }^{5, \mathrm{~d}}$ \\ ${ }^{1}$ LIP, Av. Prof. Gama Pinto, 2, 1649-003 Lisbon, Portugal \\ 2 IST University of Lisbon, Av. Rovisco Pais 1, 1049-001 Lisbon, Portugal \\ 3 Theoretical Physics Department, CERN, 1211 Geneva 23, Switzerland \\ ${ }^{4}$ Lawrence Berkeley National Laboratory, Berkeley, CA 94720, USA \\ 5 Institute of Particle Physics, Central China Normal University, Wuhan 430079, China
}

\begin{abstract}
Using a novel observable that relies on the momentum difference of the two most energetic subjets within a jet $\Delta S_{12}$ we study the internal structure of highenergy jets simulated by several Monte Carlo event generators that implement the partonic energy-loss in a dense partonic medium. Based on inclusive jet and dijet production we demonstrate that $\Delta S_{12}$ is an effective tool to discriminate between different models of jet modifications over a broad kinematic range. The new quantity, while preserving the collinear and infrared safety of modern jet algorithms, it is experimentally attractive because of its inherent resilience against backgrounds of heavy-ion collisions.
\end{abstract}

\section{Introduction}

Interactions of high-energy partons with a strongly coupled hot partonic medium - a quark-gluon plasma (QGP) [1-4] created in heavy-ion collisions, leading to modifications of the internal jet structure (jet quenching), was first proposed in [5] and is studied as a sensitive probe of the medium properties [6-8]. Experiments at RHIC and the LHC observed a strong suppression of high transverse momentum particle yields [9-15], suppression of inclusive and semi-inclusive yields of fully reconstructed jets [16-20], and, more recently, the internal structure of the jets [21-25] for detailed studies of jet quenching. However, in all these measurements the treatment of the background originating from the copiously produced particles not associated to hard scatterings poses an experimental challenge for precise and unbiased measurements. Previous works that addressed the effect of filtering

\footnotetext{
a e-mail: liliana@lip.pt

be-mail: guilherme.milhano@ist.utl.pt

c e-mail: mploskon@lbl.gov

d e-mail: xiaoming.zhang@cern.ch
}

on subjet analysis [26] and the recent analytic calculations of the momentum distributions of subjets [27-29] and groomed jet mass distributions [30-32] that were recently measured in proton-proton collisions [33,34], provide a strong motivation for novel studies. In this writeup, following previous works [35], we propose observables that are sensitive to the internal jet structure but significantly alleviate the difficulties associated to the effects of the background. Our approach is attractive from the experimental point of view and obeys the theoretical requirements of the infrared and collinear safety.

While jet substructure techniques are extensively used in the high-energy $p p$ collisions [36-38] the first studies in the heavy-ion context [24,39] are recent (see [35] for a first attempt of using subjets as a phenomenological tool for jet quenching studies). In this manuscript we propose an observable that uses only the highest and next-to-highest energetic fully reconstructed subjets within a jet. This choice aims to minimize the impact of the heavy-ion background on the extracted jet properties allowing for better experimental control.

\section{Observable definition and setup}

We introduce a new jet substructure observable $\Delta S_{12}$ defined as the difference between the fractions of transverse momentum of a jet carried by its leading (hardest) and subleading (second hardest) subjets. That is

$\Delta S_{12}=z_{1}-z_{2}$,

where

$z_{i}=p_{\mathrm{T}, i} / p_{\mathrm{T}, \mathrm{jet}}$.

The subjets used to evaluate Eq. (1) are obtained as follows: 
1. For each event, reconstruct jets with the anti- $k_{\mathrm{T}}$ algorithm [40] provided by the FASTJET package [41] with radius $R$ and within pseudo-rapidity $\left|\eta_{j e t}\right|<\eta_{\max }$

2. Within each jet, find subjets by reclustering the jet components with a smaller radius parameter $R_{\mathrm{sj}}<R$. Retain the two hardest (highest- $p_{\mathrm{T}}$ ) subjets.

The subjet samples used in this study were obtained with $\eta_{\max }=2.5$ and $R=0.5$. In general, the reclustering of the jet components into subjets in step (ii) above can be carried out with a different jet algorithm from that chosen to reconstruct the jet to which they belong. We chose to use anti- $k_{\mathrm{T}}$ after assessing the discriminating power of $\Delta S_{12}$ for subjets reconstructed with different algorithms and checking its sensitivity to hadronization effects (see Sects. 4.2 and 4.4) The subjet radius parameter was set to $R_{\mathrm{sj}}=0.15$ except when assessing, in Sect. 4.2, the dependence of $\Delta S_{12}$ on $R_{\mathrm{sj}}$ (where the range $0.1<R_{\mathrm{sj}}<0.2$ was considered), and when comparing, in Sect. 4.5, with the analysis [24] (where we used $R_{\mathrm{sj}}=0.1$ ).

The bulk of soft particles produced in high-energy collisions is not a priori distinguishable from the particles produced from the hadronisation of an energetic parton shower. The presence of these background particles is the main experimental confounding factor when establishing the jet energy scale and jet energy resolution in jet quenching studies (see for example [42]). Moreover, unlike in measurements of proton-proton collisions with high event pile-up probability within the detectors, the background in heavy-ion collisions is complex. It consists of region to region fluctuations, modified particle production as compared to pp collisions, and particle correlations caused by the collective expansion of the QGP. In consequence, experimental observables at relatively low jet energies at the $\mathrm{LHC}\left(p_{\mathrm{T}}<150 \mathrm{GeV}\right)$ are prone to systematic uncertainties related to complicated multi-dimensional unfolding procedures that are susceptible to large correction factors.

The substructure observable $\Delta S_{12}$ defined in Eq. (1) has been constructed to minimize correlated background contributions. Take [43]

$\Delta S_{12}=\frac{p_{T, 1}^{\mathrm{true}}-p_{T, 2}^{\mathrm{t} r u e}}{p_{T, \mathrm{j} e t}}=\frac{\left(p_{T, 1}^{\mathrm{rec}}-\rho_{1} A_{1}\right)-\left(p_{T, 2}^{\mathrm{r} e c}-\rho_{2} A_{2}\right)}{p_{T, \mathrm{jet}}}$,

where $p_{T, i}^{\mathrm{t} r u e}$ is the true subjet momentum, $A_{i}$ is the area of a subjet, $\rho_{i}$ is the level of noise corresponding to the amount of transverse momentum added to each subjet per unit area by the background, and the $p_{T, i}^{\mathrm{rec}}$ is the experimentally reconstructed subjet momentum containing the background contribution $\rho_{i} A_{i}$. For subjets reconstructed with the same radius parameter $R_{\mathrm{sj}}$ (in our case $R_{\mathrm{sj}}=0.15$ ) with the anti- $k_{\mathrm{T}}$ algo- rithm, the corresponding active areas are necessarily very similar $A_{1} \simeq A_{2}$. In an ideal case, where $\rho_{1}=\rho_{2}$, the background term in the numerator of $\Delta S_{12}$ vanishes. For real events, where subjets sit close by, $\rho_{1}$ and $\rho_{2}$ can only differ by very localized fluctuations and thus should be on average still very similar. Thus, the background effect in the numerator of Eq. (1) is small.

A variety of observables similar to $\Delta S_{12}$ can be defined. In particular, $z_{i}$ in Eq. (2) could be redefined by replacing the denominator by the sum of the momenta of the leading and subleading subjets, such that $z_{i}=p_{\mathrm{T}, i} /\left(p_{\mathrm{T}, 1}+p_{\mathrm{T}, 2}\right)$. Although such a definition could have some welcome consequences in reducing the influence of background effects in the reconstructed jet transverse momentum $p_{T \text {,jet }}$ (denominator of Eq. 2), all information on the overall hardness of the jet fragmentation, that is the fraction of jet momentum carried by the two hardest subjets, would be neglected.

\section{Models}

To assess the potential of the proposed observable we consider a set of Monte Carlo event generators which rely on different implementations of jet quenching. This allows both for a comparison between theoretical calculations that is not limited by systematic uncertainties of the putative experimental measurement and to assess the potential of the observable as a discriminant of different modelling scenarios.

Below we provide a short description of each event generator considered in this study - Q- PYTHIA v1.0.2 [44], JEWEL v2.0.0 [45], and PYQUEN v1.5.1 [46] - emphasising only the main characteristics and details of the setup we adopted (for further details please see the corresponding references). All samples used in this work were generated for central (0-10\% most central) $\mathrm{PbPb}$ collisions at $\sqrt{s}_{N N}=2.76 \mathrm{TeV}$.

Q- PYTHIA is a modification of PYTHIA 6.4 [47] where the splitting probability in the final state parton shower is enhanced by an additional term that follows the BDMPS-Z radiation spectrum [48]. The medium is modelled by a single parameter, a local in space and time transport coefficient $\hat{q}$ that translates the averaged transverse momentum squared $\left\langle q_{\mathrm{T}}^{2}\right\rangle$ exchanged between a parton and the medium per mean free path $\lambda$ in that medium, such that $\hat{q}=\left\langle q_{T}^{2}\right\rangle / \lambda$. The time and spatial variation of $\hat{q}$ is modelled following the PQM prescription [49]. We considered two different average $\hat{q}$ values $\left(\hat{q}=1 \mathrm{GeV}^{2} \mathrm{fm}^{-1}\right.$ and $\left.\hat{q}=4 \mathrm{GeV}^{2} \mathrm{fm}^{-1}\right)$ known to capture the main jet quenching features observed in dijets [50].

JEWEL implements a description of jet evolution that takes into account both elastic and inelastic energy losses as all scatterings with the medium are described by infra-red continued leading order matrix elements for $2 \rightarrow 2$ processes. Additional medium-induced radiation is also taken into account during the jet development and can be induced 
by several coherent scatterings, as predicted by the LPM effect $[51,52]$. We kept all default settings and used the medium implementation with Bjorken expansion described in detail in [53] validated on a large set of jet quenching observables [45].

PYQUEN is a modification (afterburner) of standard PYTHIA 6.4 jet events in which both radiative and collisional accumulated energy losses are applied during the parton shower development. The former is calculated for an expanding medium within the BDMPS framework, where the angular distribution follows three simple parameterisations (small, wide and collinear angular distributions) that are used for comparison purposes. The latter is calculated in the high-momentum transfer approximation. Additional in-medium gluon radiation is added at the end of the parton shower, before hadronization. We chose the internal parameters that characterise the QGP formation expected for central $\mathrm{PbPb}$ collisions at the LHC.

While a typical Monte Carlo reference for jet production in pp collisions is constructed with PYTHIA [47] each of the models provides their own implementation and/or modifications of PYTHIA original routines and consequently their own pp reference. Therefore, when comparing the mediummodified jets with jets showering in vacuum we take the model-provided proton-proton collision equivalent.

\section{Results}

We provide examples of how $\Delta S_{12}$ can be used to discriminate between the different implementations of jet quenching (Sect. 4.1) and evaluate its sensitivity to the choice of algorithm for subjet clustering and subjet radius $R_{\mathrm{sj}}$ (Sect. 4.2). Section 4.3 illustrates how $\Delta S_{12}$ combined with a dijet analysis can be used to study jet quenching more differentially as compared to the inclusive measurements. In Sect. 4.4 we show the robustness of the results against hadronization effects. Finally, Sect. 4.5 provides an overview of the relation between $\Delta S_{12}$ and the recently explored $z_{g}$ observable in vacuum.

\section{1 $\Delta S_{12}$ as a model discriminant}

The distribution of the difference $\Delta S_{12}$ between the fractions of the jet total transverse momentum carried by the leading and subleading subjets is shown in Fig. 1.

In vacuum - Q- PYTHIA (vac) and JEWEL (vac) (top panel), and PYTHIA 6 (bottom panel) - the distribution displays a pronounced maximum for $\Delta S_{12}>0.9$ and a tail towards lower $\Delta S_{12}$ values. Medium effects in Q- PYTHIA and JEwEL (top panel) modify the $\Delta S_{12}$ distribution in incompatible directions. JEWEL enhances significantly the maximum of the distribution and mildly depletes its tail. Q- PYTHIA softens the
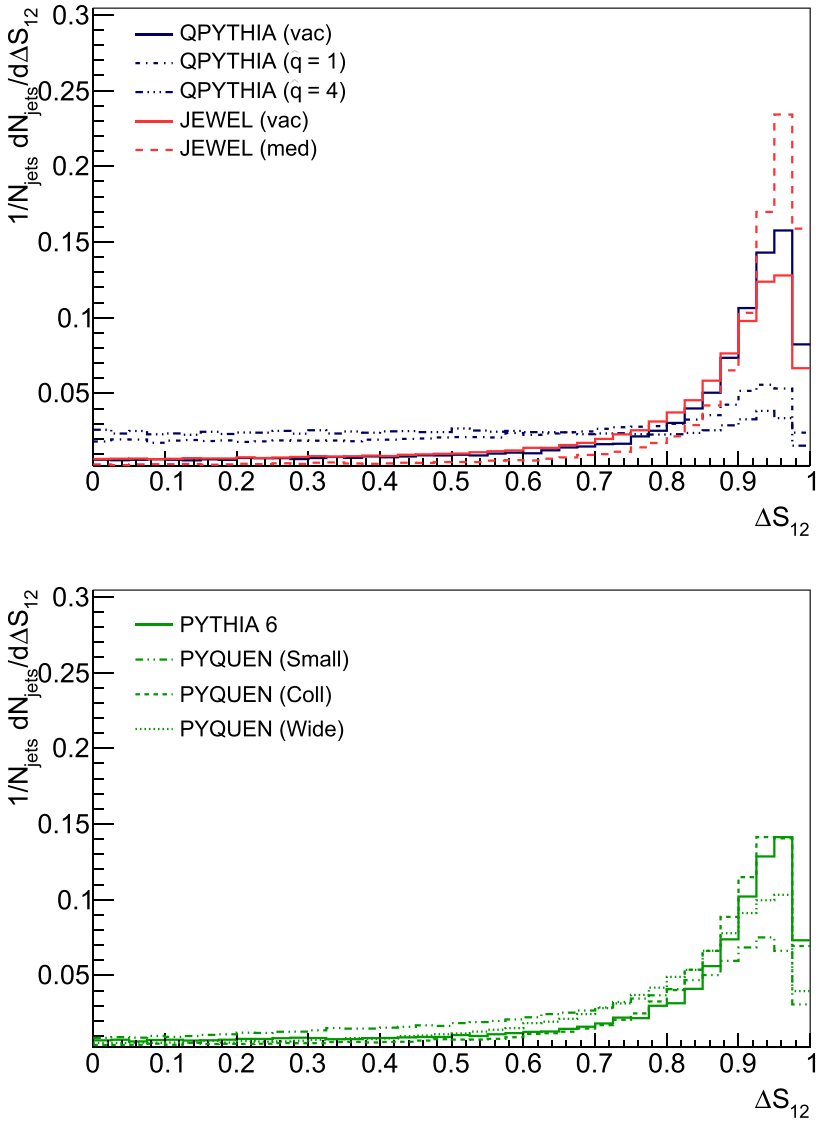

Fig. 1 Distribution of $\Delta S_{12}$ for $R_{\mathrm{sj}}=0.15$ anti- $k_{\mathrm{T}}$ subjets within $R=0.5$ anti- $k_{\mathrm{T}}$ jets with $p_{\mathrm{T}}>150 \mathrm{GeV} / c$

peak at high $\Delta S_{12}$ and produces a flat tail towards values of $\Delta S_{12} \leq 0.7$ with the effects more pronounced for increasing $\hat{q}$. These observations are consistent with a collimation of jets in JEWEL and broadening in Q- PYTHIA as compared to their vacuum references. PYQUEN (Coll) (bottom panel) modifies the $\Delta S_{12}$ similarly to JEWEL, PYQUEN (Small) gives a distribution with features resembling those found for Q- PYTHIA, and PYQUEN (Wide) displays an intermediate behaviour.

While the above features directly reflect the behaviour of the $z_{1}$ and $z_{2}$ distributions (see appendix A), we emphasise that, from the experimental point of view, studies of $\Delta S_{12}$ are more attractive as compared to the individual $z_{i}$ distributions since the difference $z_{1}-z_{2}$ removes, by construction, a large fraction of the correlated background. Although the effect of the uncorrelated background is enhanced in $\Delta S_{12}$ ratio with respect to $z_{2}$ on a jet-by-jet basis, we found that this effect is small when taking the integrated/inclusive distributions, and subsequently their moments. Moreover, $\Delta S_{12}$ is more robust for low momentum jets for which $z_{2}$ becomes gradually (with decreasing $p_{\mathrm{T}}$ ) dominated by background particles. $\mathrm{u}$

To further expose the differences among models we now turn our focus to the quartiles of the $\Delta S_{12}$ distribution, considering $\mathrm{med} / \mathrm{vac}$ ratios $\mathcal{R}$, where med refers to calculations 


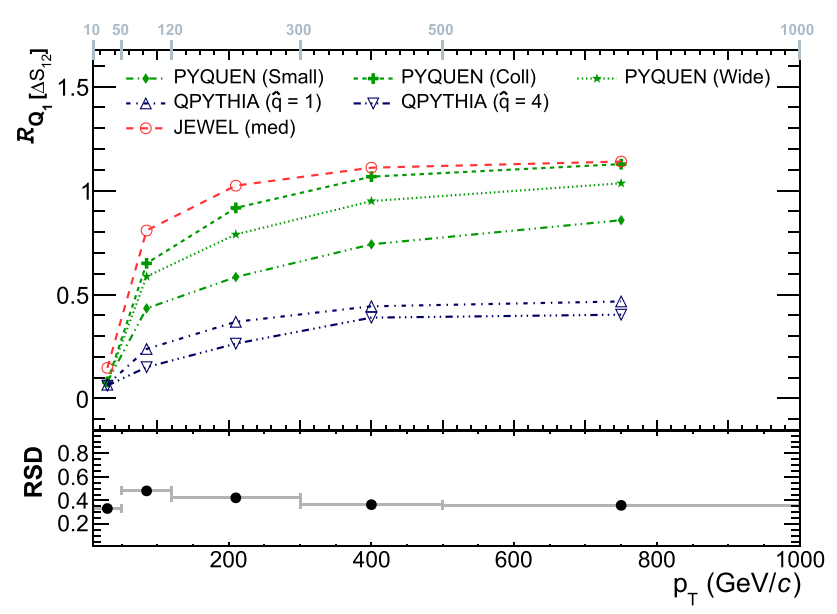

Fig. 2 Top panel: $\mathcal{R}_{\mathrm{Q}_{1}\left[\Delta S_{12}\right]}$ as a function of $p_{\mathrm{T}}^{\text {jet }}$; bottom panel: RSD of $\mathcal{R}_{Q_{1}\left[\Delta S_{12}\right]}$ as a function of $p_{\mathrm{T}}^{\text {jet }}$. The edges of the five considered jet $p_{\mathrm{T}}$ bins $(10-50,50-120,120-300,300-500,500-1000 \mathrm{GeV} / c)$ are shown on the top of the figure

including jet quenching effects and $v a c$ to the corresponding model specific no-quenching baseline

$\mathcal{R}_{\mathrm{Q}_{\mathrm{i}}\left[\Delta S_{12}\right]}=\frac{\mathrm{Q}_{\mathrm{i}}\left[\Delta S_{12}\right]^{\text {med }}}{\mathrm{Q}_{\mathrm{i}}\left[\Delta S_{12}\right]^{\text {vac }}}$

While the med/vac ratio of the medians of the $\Delta S_{12}$ distributions as a function of $p_{\mathrm{T}}^{\text {jet }}$ display a clear evolution and discrimination power among the models (data not shown), we find that to characterise the modifications to the subjet structure for models that show jet collimation - JEWEL and PYQUEN (Coll) - the ratios of the first quartile $\left(\mathrm{Q}_{1}\right)$ of $\Delta S_{12}$ distributions is preferable. The criteria of selecting the best discriminant was made by calculating the relative standard deviation (RSD) given by the models in each $p_{\mathrm{T}}$ bin, i.e, the ratio of the standard deviation over the mean. The relative spread among the different models for each observable is thus quantified (a larger spread translates into a larger RSD) and it can be used as a guiding parameter to select the observable that maximizes the differences among jet quenching models.

The ratios $\mathcal{R}_{\mathrm{Q}_{1}\left[\Delta S_{12}\right]}$ are shown in Fig. 2, in the upper panel, while the corresponding RSD in the bottom panel of the same figure.

Here we find a clear evolution with the jet momentum for jets with $p_{\mathrm{T}}<300 \mathrm{GeV} / c$ for all models. Up to this jet $p_{\mathrm{T}}$ all models show a suppression of $\mathcal{R}_{\mathrm{Q}_{1}\left[\Delta S_{12}\right]}$ reflecting more balanced momentum sharing between the two leading subjet structures than in the vacuum references. However, at high jet $p_{\mathrm{T}}$, this observable remains fairly constant and shows a strong sensitivity to models that produce jets with a more symmetric structure, such as Q- PYTHIA and PYQUEN(Small) $\left(\mathcal{R}_{\mathrm{Q}_{1}\left[\Delta S_{12}\right]}<1\right)$ separating them well apart from PYQUEN (Wide and Coll) and JEWEL $\left(\mathcal{R}_{\mathrm{Q}_{1}\left[\Delta S_{12}\right]} \simeq 1\right)$.

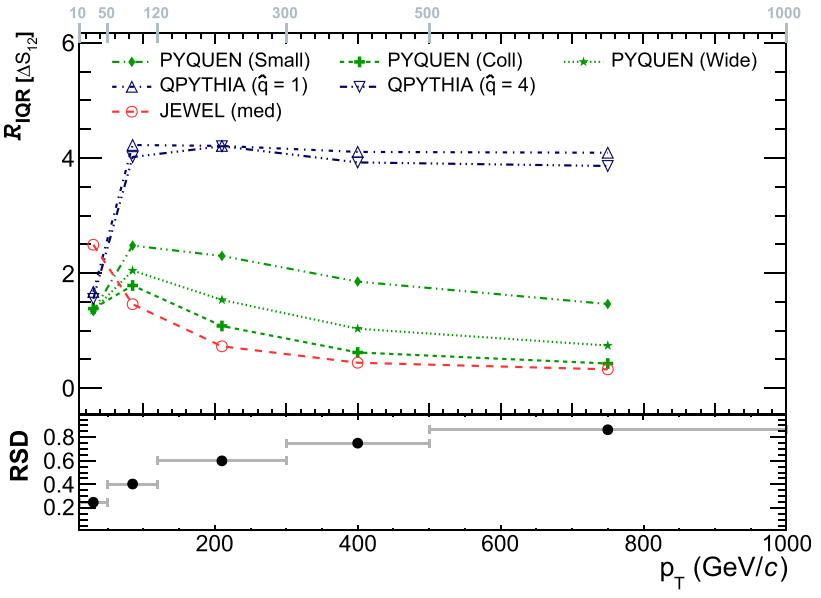

Fig. 3 Top panel: $\mathcal{R}_{\mathrm{IQR}\left[\Delta S_{12}\right]}$ as a function of $p_{\mathrm{T}}^{\text {jet }}$; bottom panel: RSD of $\mathcal{R}_{\mathrm{IQR}\left[\Delta S_{12}\right]}$ as a function of $p_{\mathrm{T}}^{\text {jet }}$. The edges of the five considered jet $p_{\mathrm{T}}$ bins $(10-50,50-120,120-300,300-500,500-1000 \mathrm{GeV} / c)$ are shown on the top of the figure

Further, we find that the interquartile range IQR $=Q_{3}-$ $\mathrm{Q}_{1}$, that characterises the width of the $\Delta S_{12}$ distribution, gives additional information. Figure 3 shows $\mathcal{R}_{\mathrm{IQR}}\left[\Delta S_{12}\right]$ as a function of jet transverse momentum for the different quenching models with the corresponding RSD calculated in each $p_{\mathrm{T}}$ bin. Here again, models that result in jet collimation, characterised by a similar or narrower $\Delta S_{12}$ distribution than its vacuum reference $\left(\mathcal{R}_{\mathrm{IQR}\left[\Delta S_{12}\right]} \leq 1\right)$, are clearly separated from those that broaden the jet, where $\Delta S_{12}$ is typically broader with respect to the vacuum reference $\left(\mathcal{R}_{\text {IQR }\left[\Delta S_{12}\right]}>1\right)$.

Moreover, for Q- PyTHIA, Jewel and PyQuen(Coll), $\mathcal{R}_{\mathrm{IQR}\left[\Delta S_{12}\right]}$ converges quickly to a constant value with increasing jet $p_{\mathrm{T}}$. Importantly, it also allows to better discriminate between the two models that destroy the vacuum subjet asymmetry: while Q- PYTHIA is well separated from its vacuum reference for all $p_{\mathrm{T}}>100 \mathrm{GeV} / c$, PyQueN(Small) evolves slowly towards more asymmetric jets with increasing $p_{\mathrm{T}}$. Thus, $\mathcal{R}_{\mathrm{IQR}}\left[\Delta S_{12}\right]$ provides relevant complementary information to identify the main characteristics of jet quenching within specific models, in particular for jets with $100<p_{\mathrm{T}}<200 \mathrm{GeV} / c$, where the first quartile $Q_{1}$ of the $\Delta S_{12}$ is suppressed with respect to the vacuum reference in all models. Comparing the RSD of $\mathcal{R}_{\mathrm{Q} 1\left[\Delta S_{12}\right]}$ and $\mathcal{R}_{\mathrm{QQR}\left[\Delta S_{12}\right]}$ we find that for low $p_{\mathrm{T}}$ jets (jets with $\left.p_{\mathrm{T}}<120 \mathrm{GeV} / c\right)$, the $\mathcal{R}_{\mathrm{Q} 1\left[\Delta S_{12}\right]}$ has a higher discrimination power while $\mathcal{R}_{\mathrm{IQR}}\left[\Delta S_{12}\right]$ is preferable for higher $p_{\mathrm{T}}$ jets. Nonetheless, it should be noted that IQR is also more sensitive to hadronization effects (see Sect. 4.4)

We also investigated the evolution of $\Delta S_{12}$ with the relative distance $\Delta R_{\text {subjet }}$ in $(\eta, \phi)$ space between the leading and subleading subjets, in particular of the med/vac of its 


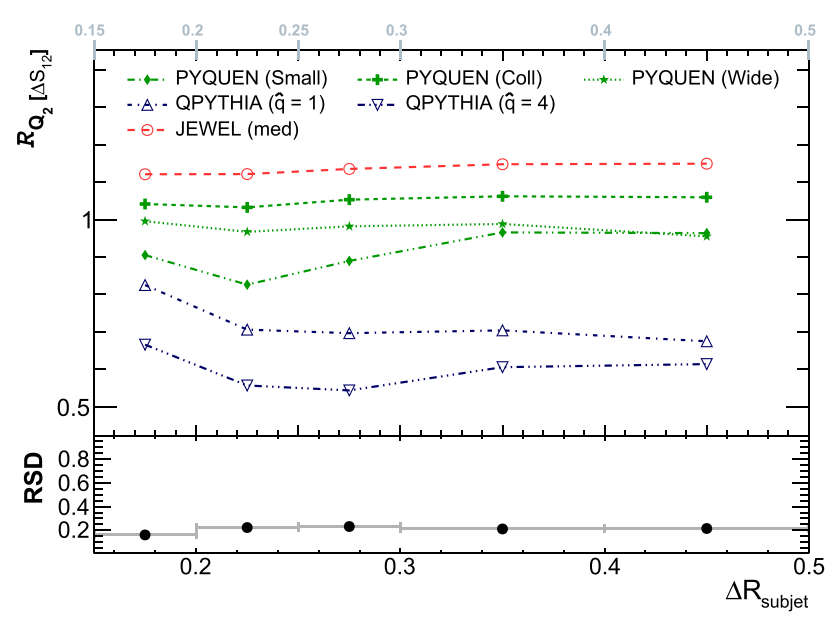

Fig. 4 Top panel: $\mathcal{R}_{\mathrm{Q}_{2}\left[\Delta S_{12}\right]}$ as a function of $\Delta R_{\text {subjet }}$; bottom panel: RSD of $\mathcal{R}_{Q_{2}\left[\Delta S_{12}\right]}$ as a function of $\Delta R_{\text {subjet }}$. The edges of the five considered $\Delta R_{\text {subjet }}$ bins: $(0.15-0.2 ; 0.2-0.25,0.25-0.3 ; 0.3-0.4 ; 0.4-$ $0.5)$ are shown on the top of the figure

median value $Q_{2}$ and interquartile range IQR. The corresponding RSD are calculated in each $\Delta R_{\text {subjet }}$ bin.

The median ratio (Fig. 4) shows a clear separation between models - Q- PYTHIA and PYQUEN(Small) - that broaden the jet structure. In Q- PYTHIA the two leading subjets become more symmetric with increasing $\Delta R_{\text {subjet }}$ (the ratio is below one and decreases). The same behaviour is seen for PYQuEN(Small) up to $\Delta R_{\text {subjet }}=0.25$, but interestingly, vacuum-like behaviour is recovered for larger separations. In contrast, the median ratio in JEWEL and PYQUEN(Coll) show a dependence on $\Delta R_{\text {subjet }}$ similar to their vacuum references with the ratio nearly independent of the distance between the two leading subjets. The large reduction of the interquartile range $\left(\mathcal{R}_{\mathrm{IQR}\left[\Delta S_{12}\right]}<0.5\right)$ for all $\Delta R_{\text {subjet }}$ observed (Fig. 5) in these models provides another clear signature of the jet collimation effect.

From the RSD values the interquartile ratio allows to have a wider spread between the models, although the transition from PYQUEN(Small) to vacuum-like behaviour is more noticeable through the median ratio.

\subsection{Dependence on the choice of subjet clustering algorithm and subjet radius}

To investigate the dependence of the proposed observable and its sensitivity to the effects of jet quenching we varied the subjet reconstruction algorithm as well as the subjet radius parameter $R_{\mathrm{sj}}<R$.

We find no significant differences in $\Delta S_{12}$ subjet distributions when changing the clustering algorithm from anti- $k_{\mathrm{T}}$ [40] to $k_{\mathrm{T}}$ [54] or Cambridge-Achen (C/A) [55]. However, $\Delta R_{\text {subjet }}$ depends, by construction, on the reconstruction algorithm. Figure 6 shows the med/vac ratio of the medians

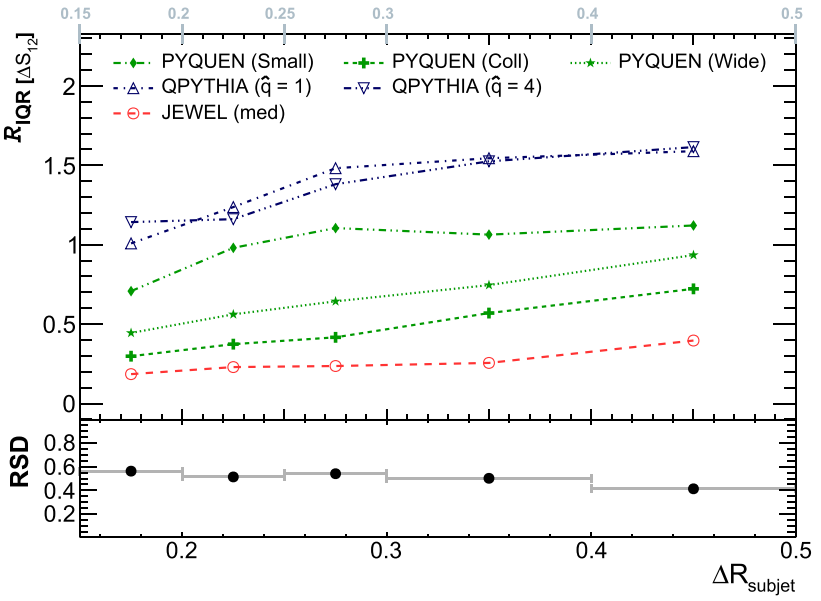

Fig. 5 Top panel: $\mathcal{R}_{\mathrm{IQR}\left[\Delta S_{12}\right]}$ as a function of $\Delta R_{\text {subjet }}$; bottom panel: RSD of $\mathcal{R}_{\mathrm{IQR}\left[\Delta S_{12}\right]}$ as a function of $\Delta R_{\text {subjet }}$. The edges of the five considered $\Delta R_{\text {subjet }}$ bins: $(0.15-0.2 ; 0.2-0.25,0.25-0.3 ; 0.3-0.4 ; 0.4-$ $0.5)$ are shown on the top of the figure

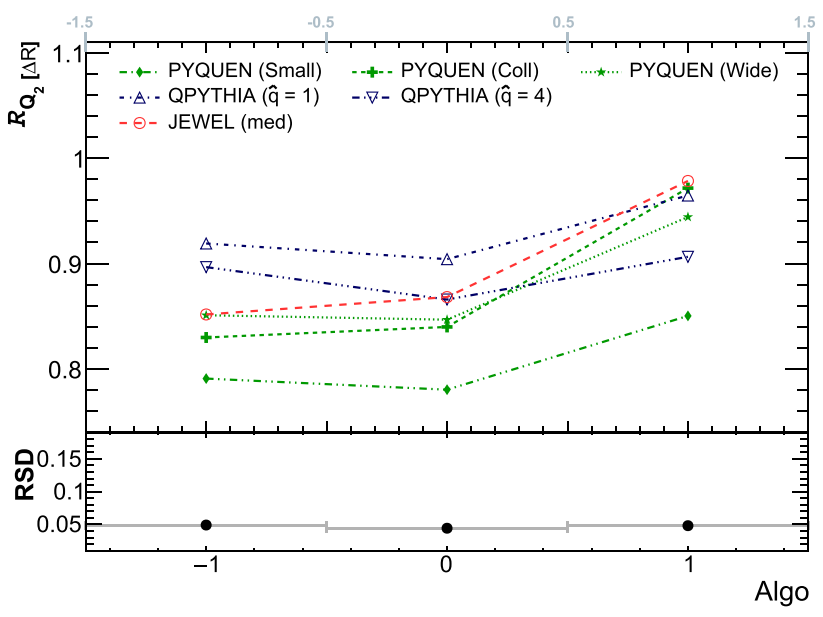

Fig. 6 Evolution of the med/vac ratio of the medians of $\Delta R$ distributions $\mathcal{R}_{Q_{2}[\Delta R]}$ (top panel) and corresponding RSD (bottom panel) with the subjet reconstruction algorithm with radius of $R_{\mathrm{sj}}=0.15$ for anti- $k_{\mathrm{T}}$ jets with $p_{\mathrm{T}}>150 \mathrm{GeV} / c$

of $\Delta R_{\text {subjet }}$ distributions for different models for $p_{\mathrm{T}}>150$ $\mathrm{GeV} / c$ jets with the subjet radius set, as before, to $R_{\mathrm{sj}}=$ 0.15 , and the corresponding RSD for completeness. The integer values $-1,0,1$ on the $\mathrm{x}$-axis correspond, respectively, to anti- $k_{\mathrm{T}}, \mathrm{C} / \mathrm{A}$, and $k_{\mathrm{T}}$. Despite the finite differences between clustering algorithms, we find that the power of discrimination between the different models is largely independent of the choice of the algorithm. This observation, together with the results obtained in Sect. 4.4, where we study the effect of different hadronization models on the reconstructed subjets, allow us to conclude that the anti- $k_{\mathrm{T}}$ algorithm provides the most promising option when optimising for jet quenching effects. We therefore adopt this clustering algorithm as the standard setting for the remainder of this work. 


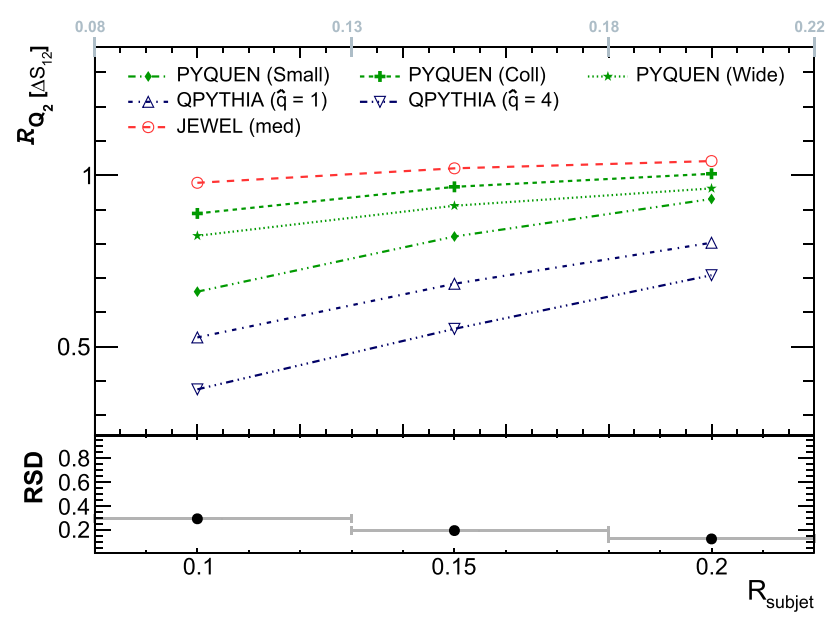

Fig. 7 Evolution of the med/vac ratio of the medians of $\Delta S_{12}$ distributions $\mathcal{R}_{\mathrm{Q}_{2}\left[\Delta S_{12}\right]}$ (top panel) and corresponding RSD (bottom panel) with the subjet radius $R_{\mathrm{Sj}}$ for anti- $k_{\mathrm{T}}$ jets with $p_{\mathrm{T}}>150 \mathrm{GeV} / c$

Figure 7 shows the dependence on $R_{\mathrm{sj}}$ of the med/vac ratio of the medians of $\Delta S_{12}$ distributions for subjets reconstructed with the anti- $k_{\mathrm{T}}$ algorithm. The RSD is also shown in the bottom panel, now calculated for each algorithm separately. Here, we find that an increased discrimination between models resulting in jet collimation and the models preferring jet broadening is achieved with $R_{\mathrm{sj}} \in[0.1 ; 0.15]$. Further, we find a clear difference in the energy distribution inside the jet that results from the different models. On the one hand, Q- PYTHIA and PYQUEN(Small) increase the leading subjet $p_{\mathrm{T}}$ when the subjet radius is increased, indicating broadening of the jet structure. On the other hand, in the models that produce collimated jets by medium effects, the energy in the leading subjet is nearly independent of the chosen subjet radius as it is highly concentrated close to the jet core.

\subsection{Subjets in dijet pairs}

In a back-to-back dijet pair propagating through the QGP, the sub-leading jet has typically lost more energy than its leading partner [56]. This quenching asymmetry can be combined with $\Delta S_{12}$ to experimentally further constrain the nature of jet quenching. We have performed an analysis of dijet pairs with $R=0.5$ anti- $k_{\mathrm{T}}$ jets within with $\left|\eta_{j e t}\right|<2$ where the leading jet was required to have $p_{\mathrm{T}}>120 \mathrm{GeV} / c$ and the recoil jet $p_{\mathrm{T}}>50 \mathrm{GeV} / c$. The jets in the pair were required to be separated in azimuth by at least $5 / 6 \pi$. The $\mathrm{med} / \mathrm{vac}$ ratios of medians of the $\Delta S_{12}$ distribution, $\mathcal{R}_{\mathrm{Q}_{2}\left[\Delta S_{12}\right]}$, as a function of $x_{J}=p_{\mathrm{T}}^{\text {recoil jet }} / p_{\mathrm{T}}^{\text {leading jet }}$ are shown in Fig. 8. The upper figures show results for leading jets and the bottom figures those for recoil jets. Again, the upper panels of each figure show the evolution of all models and the bottom panels the corresponding spread quantified through the RSD calculated in each asymmetry bin.
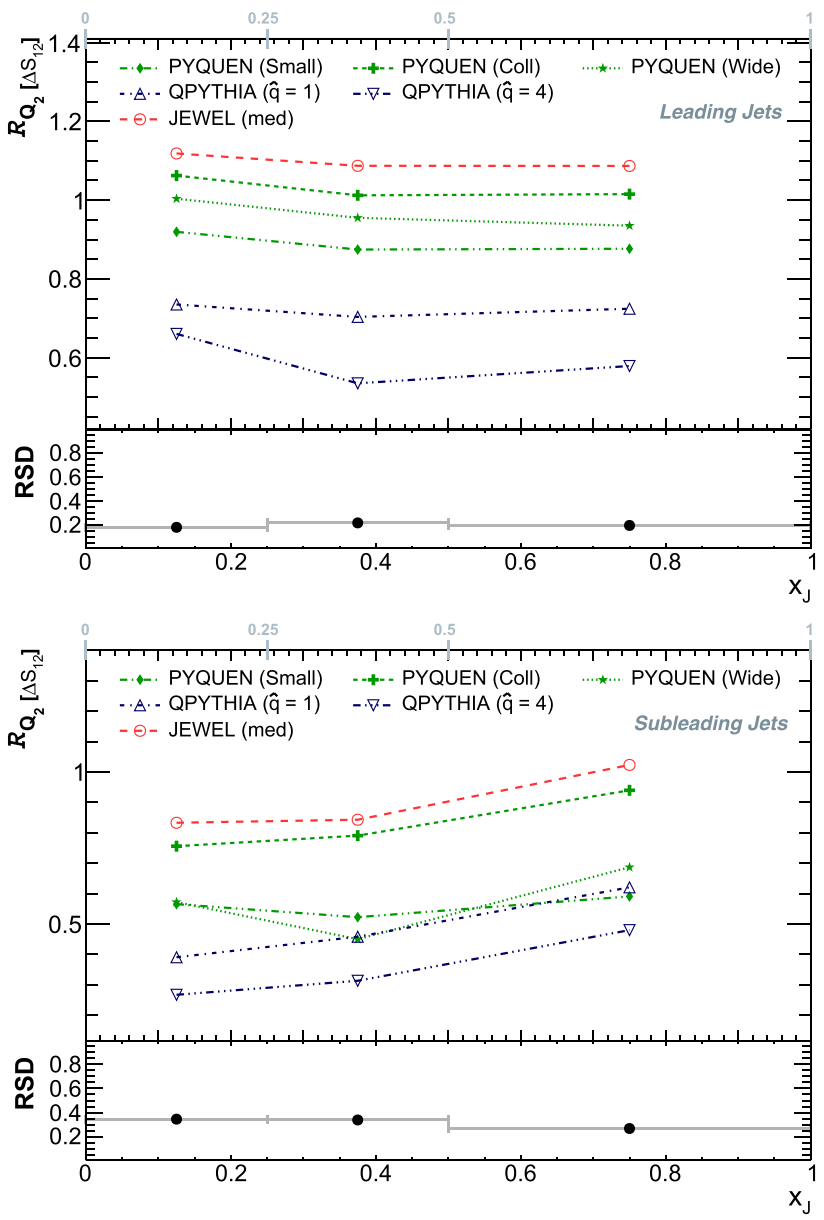

Fig. $8 \mathcal{R}_{Q_{2}\left[\Delta S_{12}\right]}$ as a function of $x_{J}$ for (top figure) leading jets and (bottom figure) recoil jets in dijet pairs. The edges of the three considered $x_{J}$ bins $(0-0.25 ; 0.25-0.5 ; 0.5-1)$ are shown on the top of the figure. The bottom panel of each figure show the corresponding RSD

Models that collimate jets towards their core - JEWEL and PYQUEN (Coll) - display leading jets with a (slightly) enhanced asymmetric subjet momentum balance as compared to their vacuum references, while the recoil jets have a more balanced subjet momentum distribution than in vacuum. In contrast, in models that broaden the jet structure Q- PYTHIA, PyQUEN (Small), and to a more limited extent PYQUEN (Wide) - both leading and recoil jets have a more balanced subjet momentum distribution than in vacuum with the modification significantly stronger for recoil jets. In all cases, the leading jet is modified independently of the pair asymmetry, while the momentum sharing between subjets becomes increasingly balanced (with respect to vacuum) with increasing dijet asymmetry (decreasing $x_{J}$ ) for recoil jets.

Similar findings are also present in the ratio of the interquartile range of the distributions, for both leading and recoil jets, shown in Fig. 9. However, differences between leading jets are more noticeable through the asymmetry of the $\Delta S_{12}$ distribution while recoil jets show a larger spread among models through $\mathcal{R}_{\mathrm{Q}_{2}\left[\Delta S_{12}\right]}$ 

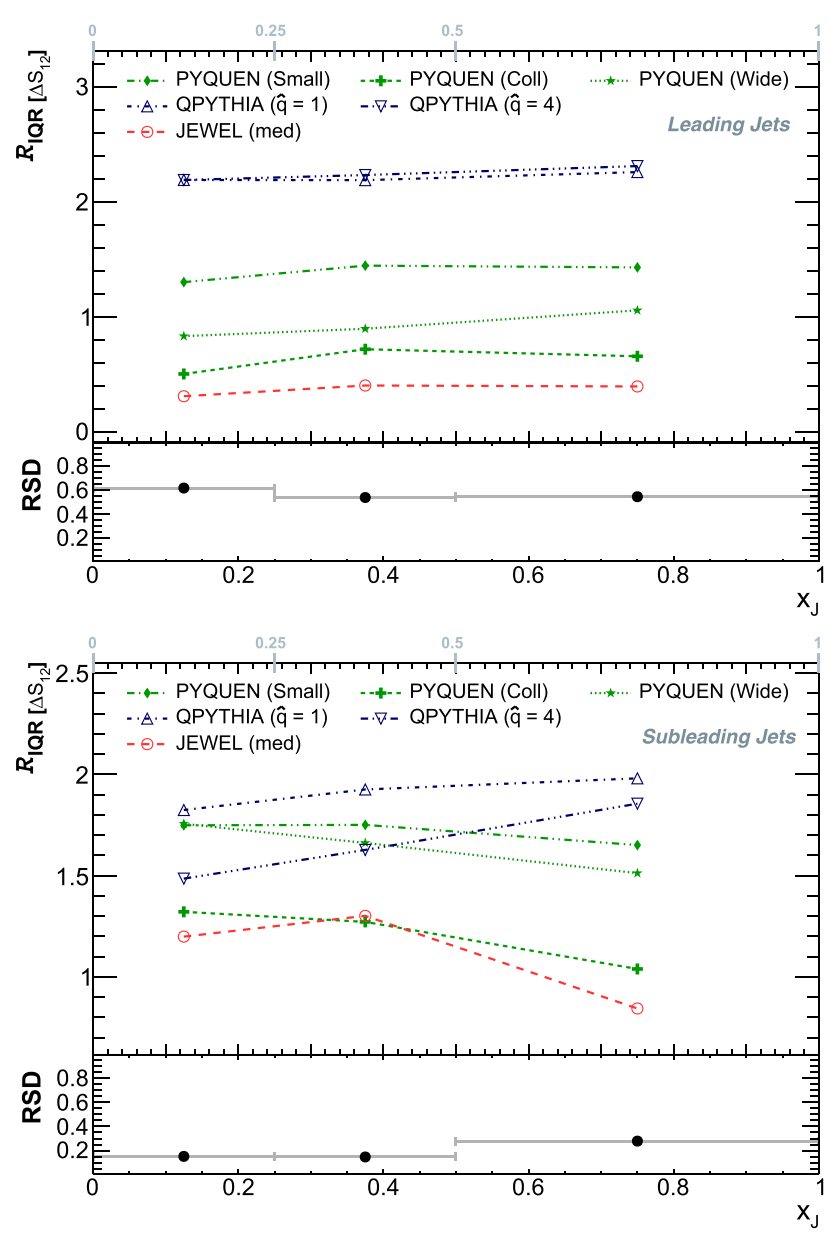

Fig. $9 \mathcal{R}_{\mathrm{IRQ}\left[\Delta S_{12}\right]}$ as a function of $x_{J}$ for (top figure) leading jets and (bottom figure) recoil jets in dijet pairs. The edges of the three considered $x_{J}$ bins $(0-0.25 ; 0.25-0.5 ; 0.5-1)$ are shown on the top of the figure. The bottom panel of each figure show the corresponding RSD

All these observations are consistent with the findings from Sect. 4.1, Fig. 2, where jets below $p_{\mathrm{T}}=200 \mathrm{GeV} / c$ always have a $\Delta S_{12}$ that is smaller than its vacuum reference. This is the preferred kinematic region for the recoil jet in unbalanced dijet systems. Moreover, $\mathcal{R}_{\mathrm{Q}_{1}\left[\Delta S_{12}\right]}$ is fairly constant for jets above $p_{\mathrm{T}}=200 \mathrm{GeV} / c$, where the leading jet (and recoil for balanced dijet systems) typically comes.

\subsection{Hadronization effects on the reconstructed subjets}

Small radii jets are known to be more sensitive to hadronization effects[57]. For this reason, we investigate the role of different hadronization models in the distributions that were presented so far by using both PYTHIA 8 and HERWIG 7 [58,59]. The former is based solely on the Lund string fragmentation framework [60] while the later applies a cluster model [61] to hadronize the resulting partonic final state to produce hadrons. Although such study is not ideal to accurately assess the uncertainties induced by hadronization effects, including in-medium hadronization modifica- tions [62], it can provide an estimate of the robustness of the proposed observable, $\Delta S_{12}$.

We have found that $\mathrm{Q}_{2}\left[\Delta S_{12}\right]$ is almost insensitive to the hadronization model, with relative differences (taking PYTHIA 8 as reference) smaller than $1 \%$ for any choice of subjet radius or clustering algorithm and jets with a transverse momentum $p_{T, \text { jet }}>100 \mathrm{GeV} / c$. For low momentum jets $\left(p_{T, \text { jet }}<100 \mathrm{GeV} / c\right)$, this difference goes up to $2 \%$ for $R_{\text {subjet }}=0.15$ and $10 \%$ for $R_{\text {subjet }}=0.1$.

As for the first quartile of the distribution, $\mathrm{Q}_{1}\left[\Delta S_{12}\right]$, the relative change of HERWIG 7 with respect to PYTHIA 8 is $\sim[2-5] \%$ for anti- $k_{\mathrm{T}}$ subjets with $R_{\text {subjet }} \leq 0.15$. Any other choice of clustering algorithm or subjet radius provide a relative difference of $\sim[5-10] \%$ independently of the jet transverse momentum.

Finally, the interquartile range, IQR $\left[\Delta S_{12}\right]$ that is able to provide, in general, a larger dispersion between the jet quenching models, is also able to discriminate more among hadronization models. The relative change in low momentum jets $\left(p_{T, \text { jet }}<100 \mathrm{GeV} / c\right)$ between the interquartile range provided by the two Monte Carlo event generators is around [4-10]\% for any clustering algorithm and subjets reconstructed with $R_{\text {subjet }} \leq 0.15$. For $R_{\text {subjet }}=0.2$, this change increases to $17 \%$, independently of the clustering algorithm. In high momentum jets $\left(p_{T, \text { jet }}>250 \mathrm{GeV} / c\right)$ the relative difference is around $[20-40] \%$. The lower bracketing is constantly observed for anti- $k_{\mathrm{T}}$ and small radius subjets while the upper bracketing occurs for $k_{\mathrm{T}}$ and large radius subjets. For the chosen parameters of this manuscript (anti- $k_{\mathrm{T}}$ subjets with $R_{\text {subjet }}=0.15$ ), the relative change is $\sim 25 \%$ for any jet with $p_{T, \text { jet }}>100 \mathrm{GeV} / c$.

The general large sensitivity of the interquartile range to the choice of the hadronization model comes from the fact that this observable is designed to promote the tails of the distributions. While it is the preferable region to tag energy loss modifications imprinted on the jet, it is also the region dominated by a fragmentation pattern that promotes the existence of one $\left(\Delta S_{12} \sim 1\right)$ or two $\left(\Delta S_{12} \sim 2\right)$ subjets mainly composed by very soft particles. Any modification on the hadronization mechanism would imply a stronger deviation on both $\mathrm{Q}_{1}\left[\Delta S_{12}\right]$ (as observed from the increase of the relative differences with respect to $\left.\mathrm{Q}_{2}\left[\Delta S_{12}\right]\right)$ and $\mathrm{Q}_{3}\left[\Delta S_{12}\right]$.

These observations validate our choice of using reclustered anti- $k_{\mathrm{T}}$ subjets with $R_{\text {subjet }}=0.15$ as to maximize jet quenching phenomena with respect to hadronization effects.

\subsection{Sub-jet momentum fraction $z_{g}$ and $\Delta S_{12}$}

Recent studies of the momentum fraction $z_{g}$ in jets [24] prompt for a comparison of $z_{g}$ with $\Delta S_{12}$. We have performed an analysis of (vacuum) PYTHIA jets with $p_{\mathrm{T}}>150 \mathrm{GeV} / c$ using settings of the SOFT DROP algorithm $[63,64]$ as in [24]. Figure 10 shows the $z_{g}$ as a function of $\Delta S_{12}$ for two 

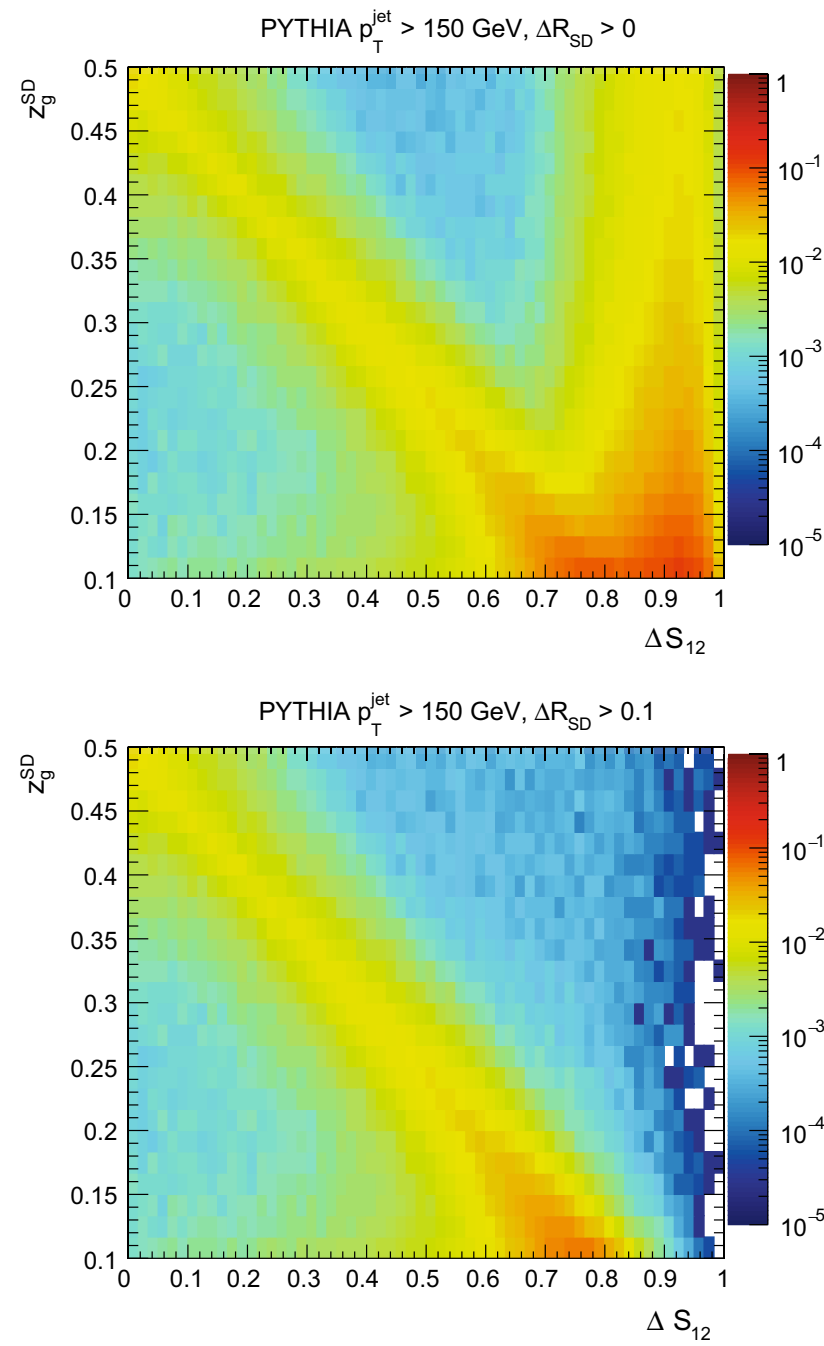

Fig. 10 Subjet momentum fraction $z_{g}^{S D}$ reconstructed using the SoFT DROP algorithm as a function of $\Delta S_{12}$. Upper panel: distribution for all jets with $p_{\mathrm{T}}>150 \mathrm{GeV} / c$. Lower panel: distribution for jets for which subjets used for calculating $z_{g}$ are separated by a distance of $\Delta R>0.1$

cases: one, where all jets where used; and a second, where jets with $\Delta R_{\mathrm{sj}}<0.1$ between the subjets used to calculate $z_{g}$ are discarded. $\Delta S_{12}$ was calculated with $R_{\text {subjet }}=0.1$ in both cases. We find a strong correlation between $\Delta S_{12}$ and the calculation of $z_{g}$ when using the $\Delta R_{\mathrm{sj}}$ cut as in [24]. Without the cut on $\Delta R_{\mathrm{sj}}$ the distributions have two dominating structures. One is the diagonal, but the other is largely independent of the $\Delta S_{12}$ at $\Delta S_{12}>0.8$.

A comment on the differences of behaviour between these two observables in the presence of a QGP is in order. One of the proposed explanations [65] for the observed modification of the $z_{g}$ distribution in nucleus-nucleus collisions relies on the ability of subjets to collect contributions from the QGP. As argued in that work, this QGP backreaction process implies a distinctive increase in size (measured girth) of the subjets. Since in $\Delta S_{12}$ we explicitly impose, by specifying a radius parameter $R_{\mathrm{sj}}$ for the subjet reconstruction, a
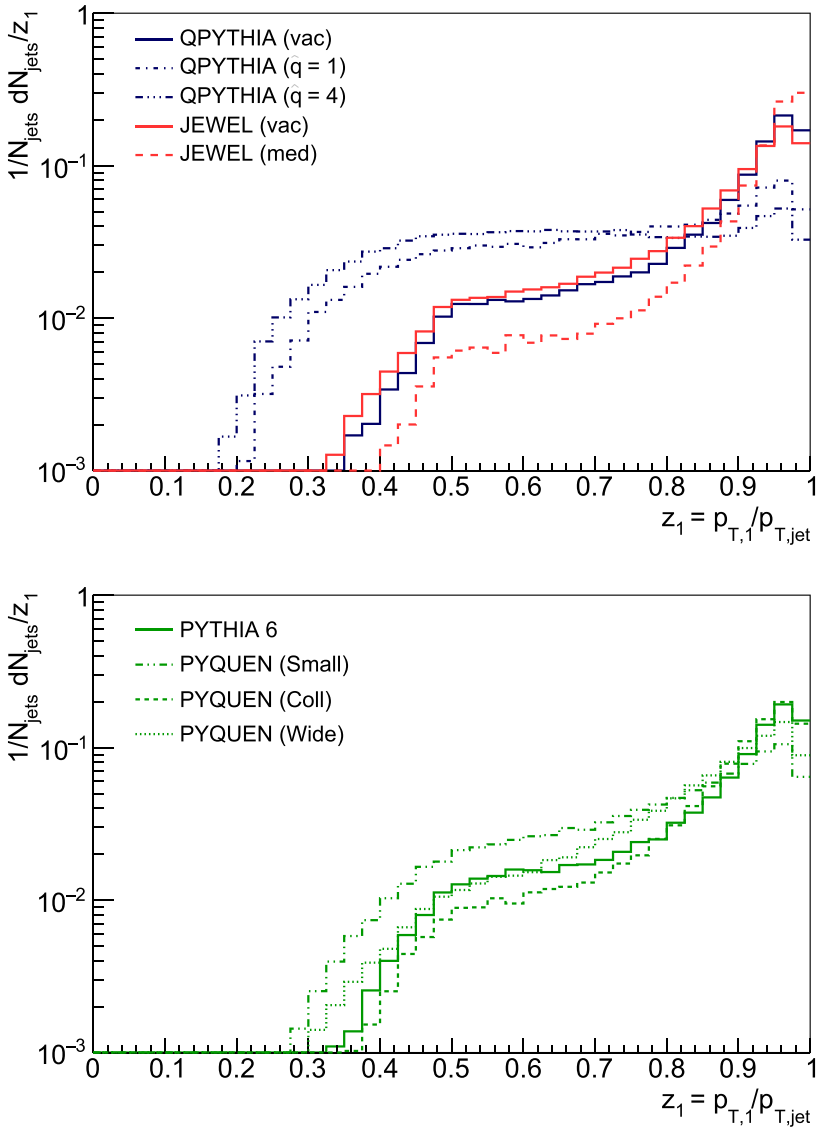

Fig. 11 The fraction of transverse momenta of anti- $k_{\mathrm{T}} R=0.5$ jets with $p_{\mathrm{T}}>150 \mathrm{GeV} / c$ carried by the leading anti- $k_{\mathrm{T}}$ subjet reconstructed with $R_{\mathrm{sj}}=0.15$

size for the subjets, contributions from the QGP to both subjets will be of the same order, and thus, will cancel in $\Delta S_{12}$. This makes $\Delta S_{12}$ and $z_{g}$, well correlated in vacuum, complementary observables in the presence of a QGP that can be used to disentangle the role of QGP backreaction from other dynamical processes that conceivably modify the jet substructure.

\section{Discussion}

JEWEL. Results from JEWEL are consistent with a jet collimation effect, i.e., most of the radiation is transported outside of the cone leaving the energy core of the jet almost un-modified with respect to the vacuum reference but in a narrower region of phase space. We note, that in this analysis we have used JEWEL in its "recoil-off" mode which discards the medium partons that interacted with the jet. In this way, the results are independent of the medium-response, whose impact was recently analysed in [65]. As a consequence, the $\Delta S_{12}$ is closer to unity for the medium modified jets and it does not change with the chosen subjet subjet radius. Moreover, the comparison of properties of the leading and sub- 

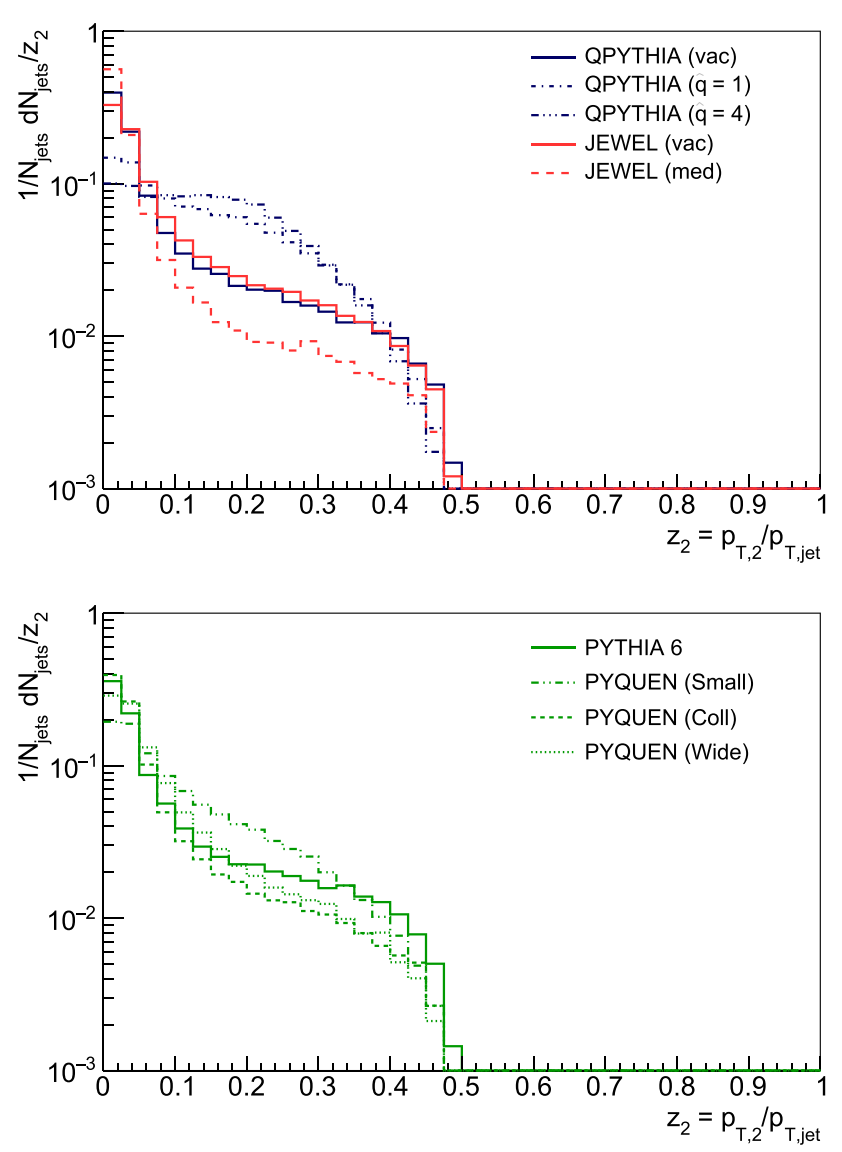

Fig. 12 The fraction of transverse momenta of anti- $k_{\mathrm{T}} R=0.5$ jets with $p_{\mathrm{T}}>150 \mathrm{GeV} / c$ carried by the subleading anti- $k_{\mathrm{T}}$ subjet reconstructed with $R_{\mathrm{sj}}=0.15$

leading jets from a dijet event shows that $\Delta S_{12}$ increases for the leading jets and decreases for the (more strongly mediummodified/low momentum) subleading jet. This is an exclusive characteristic of the jet collimation phenomena and/or collisional energy loss as the same kind of behaviour is observed for PYQuen(Coll). In this model, since $\theta_{\text {rad }}=0$, all the energy that is lost outside of the cone is due to elastic energy loss.

Q- PYTHIA. In Q- PYTHIA, which is as an implementation of the BDMPS-Z spectrum (without account for destructive interferences), the emission rate is enhanced according to the quenching parameter $\hat{q}$ leading to a large modifications of the jet inner core. As a consequence, the distribution with a maximum for $\Delta S_{12}>0.9$ in vacuum shows a large tail to lower values due to in-medium interactions due to softening of the subjet spectrum (including the leading subjet). Such effect is visible for both leading and subleading jets. Moreover, the medium-induced gluon radiation is evenly distributed in phase space up to very large distances as $\Delta S_{12}$ mean value is constantly below the vacuum reference without a significant change for $\Delta R_{\text {subjet }}>0.2$.
PYQUEN. PYQUEN considerations are centred around three angular distributions for the in-medium radiation spectrum. For PYQuEN(Small) the finite angle of the radiation $\left(\theta<5^{\circ}\right)$ enhances the substructure and the impact on $\Delta S_{12}$ is qualitatively similar to Q- PYTHIA. Nonetheless, a striking difference from this model with respect to Q- PYTHIA is the increasing asymmetry of the subjet structure when biasing the jet sample with $\Delta R_{\text {subjets }}>0.3$. This could be due to the fact that since the radiation is displaced at a finite angle from the leading parton, the more the second hardest subjet is reconstructed away from the jet core, the less probable is to recover the energy. As such, the energy-momentum distribution inside of the jet is located at intermediate distances from the jet core in contrast to what happens in Q- PYTHIA. A similar, but much milder modification of $\Delta S_{12}$ is observed for PYQUEN(Wide). Since gluon radiation goes as $\sim 1 / \theta$, the radiation is essentially kept near the core with few particles going to very large angles. This angular distribution is similar to the jet vacuum development, which makes this model undistinguishable from the vacuum reference for jets with a large transverse momentum and/or leading jets in dijet systems. On the other hand, the PYQUEN(Coll) mode, constrained to elastic energy loss only, affects mainly the softest jet constituents by such elastic collisions, whose energy is "absorbed" by the medium. As such, jets become more collimated, as it happens in JEWEL.

Considerations of the RSD distributions show that the $\mathcal{R}_{\mathrm{IQR}\left[\Delta S_{12}\right]}$ carries the largest discrimination power for high$p_{\mathrm{T}}$ jets, although there is an associated uncertainty of [10$25] \%$ due to hadronization effects. For low- $p_{\mathrm{T}}$ jets $\left(p_{\mathrm{T}} \lesssim\right.$ $120 \mathrm{GeV} / c$ ) and/or recoil-jets in dijet systems the use of the $\mathcal{R}_{\mathrm{Q}_{1}\left[\Delta S_{12}\right]}$ and/or the median $\mathcal{R}_{\mathrm{Q}_{2}\left[\Delta S_{12}\right]}$ may prove more advantageous, with hadronization uncertainties that are smaller than 5\% for the chosen subjet parameters.

Finally, we reiterate that inclusive or semi-inclusive measurements of nuclear modification factor(s) for jets that fall within a range of $\Delta S_{12}$ (and $\Delta R_{\text {subjets }}$ ) can provide a rather straightforward insight into the properties of jet quenching (see [35] for example).

\section{Conclusions}

We have presented observables of subjet structure that by minimizing the impact of the particle backgrounds in heavyion collisions are advantageous from the experimental point of view. At the same time, the introduced $\Delta S_{12}$ quantity preserves the collinear and infrared safety of modern jet algorithms. Using a number of Monte Carlo jet quenching models we have demonstrated that $\Delta S_{12}$ distribution and $\Delta R_{\text {subjets }}$ can be used as a sensitive tool to discriminate between different quenching mechanisms. We have shown that it is possible to use the quartiles of those distributions, together with 
the widths and/or use of dijets to make an accurate assessment of the main jet quenching characteristics, in particular, to determine the angular structure of the medium-induced gluon radiation and to investigate further the role of collisional energy loss in the in-medium shower development.

Acknowledgements The authors would like to thank to N. Armesto, M. Verweij and K. Zapp for useful discussions. This work was supported in part by the U.S. Department of Energy, Office of Science, Office of Nuclear Physics, under contract DE-AC02-05CH11231 (MP, XZ) and by the Fundação para a Ciência e Tecnologia (Portugal) under contracts CERN/FIS-NUC/0049/2015, Investigador FCT - Development Grant IF/00563/2012 (JGM) and SFRH/BPD/103196/2014 (LA).

Open Access This article is distributed under the terms of the Creative Commons Attribution 4.0 International License (http://creativecomm ons.org/licenses/by/4.0/), which permits unrestricted use, distribution, and reproduction in any medium, provided you give appropriate credit to the original author(s) and the source, provide a link to the Creative Commons license, and indicate if changes were made.

Funded by SCOAP ${ }^{3}$.

\section{Appendix: A leading and subleading subjets}

The gross features of the differences between the models have been discussed in terms of $\Delta S_{12}$ in Sect. 4.1. In this appendix we present the individual $z_{i}$ distributions only for completeness and with a limited analysis. The distribution of the fraction $z_{1}$ of the jet total transverse momentum carried by the leading subjet in jets with $p_{\mathrm{T}}>150 \mathrm{GeV} / c$ is shown in Fig. 11 for (top panel) Q- PYTHIA and JEWEL, and (bottom panel) PYQUEN with its three radiation pattern variants. The vacuum references for each model - Q- PYTHIA (vac), JEWEL (vac), and PYTHIA (for PYQUEN) - are also shown. Clearly Q- PYTHIA and JEWEL modify the $z_{1}$ distribution in incompatible directions. As noted for $\Delta S_{12}$ these observations are consistent with a collimation of jets within JEWEL and broadening in Q- PYTHIA as compared to their vacuum references. For PYQUEN (botton panel) we find a clear separation of its different parametrisations of the angular distribution of medium induced radiation. The $z_{2}$ distribution (the $p_{\mathrm{T}}$ fraction carried by the subleading subjet) shown in Fig. 12 is, by definition, limited to the $0-0.5$ interval. The differences among the $z_{2}$ distributions obtained from the different models mirror those observed for $z_{1}$. Globally, the $p_{\mathrm{T}}$ fraction $z_{2}$ carried by the subleading subjet reflects the strongly peaked $z_{1}$ distribution at large- $z$ which necessarily places the average $z_{2}$ to be below 0.1 .

\section{References}

1. H. Satz, Rept. Prog. Phys. 63, 1511 (2000). https://doi.org/10.1088/ 0034-4885/63/9/203
2. S.A. Bass, M. Gyulassy, H. Stoecker, W. Greiner, J. Phys. G25, R1 (1999). https://doi.org/10.1088/0954-3899/25/3/013

3. E.V. Shuryak, Phys. Rept. 115, 151 (1984)

4. J. Cleymans, R. Gavai, E. Suhonen, Phys. Rept. 130, 217 (1986). https://doi.org/10.1016/0370-1573(86)90169-9

5. J.D. Bjorken (1982)

6. U.A. Wiedemann, pp. 521-562 (2010). https://doi.org/10.1007/ 978-3-642-01539-7-17

7. K.M. Burke et al., Phys. Rev. C 90(1), 014909 (2014). https://doi. org/10.1103/PhysRevC.90.014909

8. Y. Mehtar-Tani, J.G. Milhano, K. Tywoniuk, Int. J. Mod. Phys. A 28, 1340013 (2013). https://doi.org/10.1142/ S0217751X13400137

9. J. Adams et al., Nucl. Phys. A 757, 102 (2005). https://doi.org/10. 1016/j.nuclphysa.2005.03.085

10. K. Adcox et al., Nucl. Phys. A 757, 184 (2005). https://doi.org/10. 1016/j.nuclphysa.2005.03.086

11. I. Arsene et al., Nucl. Phys. A 757, 1 (2005). https://doi.org/10. 1016/j.nuclphysa.2005.02.130

12. B. Back et al., Nucl. Phys. A 757, 28 (2005). https://doi.org/10. 1016/j.nuclphysa.2005.03.084

13. K. Aamodt et al., Phys. Lett. B 696, 30 (2011). https://doi.org/10. 1016/j.physletb.2010.12.020

14. K. Aamodt et al., Phys. Rev. Lett. 108, 092301 (2012). https://doi. org/10.1103/PhysRevLett.108.092301

15. S. Chatrchyan et al., Eur. Phys. J. C 72, 1945 (2012). https://doi. org/10.1140/epjc/s10052-012-1945-x

16. G. Aad et al., Phys. Rev. Lett. 105, 252303 (2010). https://doi.org/ 10.1103/PhysRevLett.105.252303

17. S. Chatrchyan et al., Phys. Rev. C 84, 024906 (2011). https://doi. org/10.1103/PhysRevC.84.024906

18. J. Adam et al., Phys. Lett. B 746, 1 (2015). https://doi.org/10.1016/ j.physletb.2015.04.039

19. L. Adamczyk et al., Phys. Rev. C 96(2), 024905 (2017). https:// doi.org/10.1103/PhysRevC.96.024905

20. J. Adam et al., JHEP 09, 170 (2015). https://doi.org/10.1007/ JHEP09(2015) 170

21. G. Aad et al., Phys. Lett. B 739, 320 (2014). https://doi.org/10. 1016/j.physletb.2014.10.065

22. S. Chatrchyan et al., Phys. Lett. B 730, 243 (2014). https://doi.org/ 10.1016/j.physletb.2014.01.042

23. S. Chatrchyan et al., JHEP 1210, 087 (2012). https://doi.org/10. 1007/JHEP10(2012)087

24. A.M. Sirunyan et al., Phys. Rev. Lett. 120(14), 142302 (2018). https://doi.org/10.1103/PhysRevLett.120.142302

25. L. Cunqueiro, Nucl. Phys. A 956, 593 (2016). https://doi.org/10. 1016/j.nuclphysa.2016.02.060

26. M. Rubin, JHEP 05, 005 (2010). https://doi.org/10.1007/ JHEP05(2010)005

27. Z.B. Kang, F. Ringer, W.J. Waalewijn, JHEP 07, 064 (2017). https:// doi.org/10.1007/JHEP07(2017)064

28. A.J. Larkoski, S. Marzani, J. Thaler, Phys. Rev. D 91(11), 111501 (2015). https://doi.org/10.1103/PhysRevD.91.111501

29. A. Larkoski, S. Marzani, J. Thaler, A. Tripathee, W. Xue, Phys. Rev. Lett. 119(13), 132003 (2017). https://doi.org/10.1103/ PhysRevLett.119.132003

30. C. Frye, A.J. Larkoski, M.D. Schwartz, K. Yan, JHEP 07, 064 (2016). https://doi.org/10.1007/JHEP07(2016)064

31. S. Marzani, L. Schunk, G. Soyez, JHEP 07, 132 (2017). https:// doi.org/10.1007/JHEP07(2017)132

32. S. Marzani, L. Schunk, G. Soyez, Eur. Phys. J. C 78(2), 96 (2018). https://doi.org/10.1140/epjc/s10052-018-5579-5

33. A.M. Sirunyan et al. (2018). https://doi.org/10.3204/ PUBDB-2018-02200

34. M. Aaboud et al. (2017). arXiv:1711.08341 
35. X. Zhang, L. Apolinário, J.G. Milhano, M. Ploskon, Nucl. Phys. A 956, 597 (2016). https://doi.org/10.1016/j.nuclphysa.2016.02.028

36. S. Chatrchyan et al., JHEP 05, 090 (2013). https://doi.org/10.1007/ JHEP05(2013)090

37. G. Aad et al., JHEP 05, 128 (2012). https://doi.org/10.1007/ JHEP05(2012) 128

38. A. Tripathee, W. Xue, A. Larkoski, S. Marzani, J. Thaler, Phys. Rev. D 96(7), 074003 (2017). https://doi.org/10.1103/PhysRevD. 96.074003

39. K. Kauder, Nucl. Part. Phys. Proc. 289-290, 137 (2017). https:// doi.org/10.1016/j.nuclphysbps.2017.05.028

40. M. Cacciari, G.P. Salam, G. Soyez, JHEP 04, 063 (2008). https:// doi.org/10.1088/1126-6708/2008/04/063

41. M. Cacciari, G.P. Salam, G. Soyez, Eur. Phys. J. C 72, 1896 (2012). https://doi.org/10.1140/epjc/s10052-012-1896-2

42. B. Abelev et al., JHEP 1203, 053 (2012). https://doi.org/10.1007/ JHEP03(2012)053

43. M. Cacciari, G.P. Salam, Phys. Lett. B 659, 119 (2008). https://doi. org/10.1016/j.physletb.2007.09.077

44. N. Armesto, L. Cunqueiro, C.A. Salgado, Eur. Phys. J. C 63, 679 (2009). https://doi.org/10.1140/epjc/s10052-009-1133-9

45. K.C. Zapp, Eur. Phys. J. C 74(2), 2762 (2014). https://doi.org/10. 1140/epjc/s10052-014-2762-1

46. I.P. Lokhtin, A.M. Snigirev, Eur. Phys. J. C 45, 211 (2006). https:// doi.org/10.1140/epjc/s2005-02426-3

47. T. Sjostrand, S. Mrenna, P.Z. Skands, JHEP 05, 026 (2006). https:// doi.org/10.1088/1126-6708/2006/05/026

48. R. Baier, Y.L. Dokshitzer, A.H. Mueller, S. Peigne, D. Schiff, Nucl. Phys. B 484, 265 (1997). https://doi.org/10.1016/ S0550-3213(96)00581-0

49. A. Dainese, C. Loizides, G. Paic, Eur. Phys. J. C 38, 461 (2005). https://doi.org/10.1140/epjc/s2004-02077-x
50. L. Apolinario, N. Armesto, L. Cunqueiro, JHEP 02, 022 (2013). https://doi.org/10.1007/JHEP02(2013)022

51. L.D. Landau, I. Pomeranchuk, Dokl. Akad. Nauk Ser. Fiz. 92, 535 (1953)

52. A.B. Migdal, Phys. Rev. 103, 1811 (1956). https://doi.org/10.1103/ PhysRev.103.1811

53. K.C. Zapp, Phys. Lett. B 735, 157 (2014). https://doi.org/10.1016/ j.physletb.2014.06.020

54. S.D. Ellis, D.E. Soper, Phys. Rev. D 48, 3160 (1993). https://doi. org/10.1103/PhysRevD.48.3160

55. Y.L. Dokshitzer, G.D. Leder, S. Moretti, B.R. Webber, JHEP 08, 001 (1997). https://doi.org/10.1088/1126-6708/1997/08/001

56. J.G. Milhano, K.C. Zapp, Eur. Phys. J. C 76(5), 288 (2016). https:// doi.org/10.1140/epjc/s10052-016-4130-9

57. M. Dasgupta, L. Magnea, G.P. Salam, JHEP 02, 055 (2008). https:// doi.org/10.1088/1126-6708/2008/02/055

58. M. Bahr et al., Eur. Phys. J. C 58, 639 (2008). https://doi.org/10. 1140/epjc/s10052-008-0798-9

59. J. Bellm et al., Eur. Phys. J. C 76(4), 196 (2016). https://doi.org/ 10.1140/epjc/s10052-016-4018-8

60. B. Andersson, G. Gustafson, G. Ingelman, T. Sjostrand, Phys. Rept. 97, 31 (1983). https://doi.org/10.1016/0370-1573(83)90080-7

61. A. Kupco, pp. 292-300 (1998)

62. A. Beraudo, J.G. Milhano, U.A. Wiedemann, Phys. Rev. C 85, 031901 (2012). https://doi.org/10.1103/PhysRevC.85.031901

63. M. Dasgupta, A. Fregoso, S. Marzani, G.P. Salam, JHEP 09, 029 (2013). https://doi.org/10.1007/JHEP09(2013)029

64. A.J. Larkoski, S. Marzani, G. Soyez, J. Thaler, JHEP 05, 146 (2014). https://doi.org/10.1007/JHEP05(2014)146

65. J.G. Milhano, U.A. Wiedemann, K.C. Zapp (2017) https://doi.org/ 10.1016/j.physletb.2018.01.029 